\title{
Égalités et inégalités dans l'activité créatrice. Durkheim, Marx et Rawls devant l'individualisme artistique
}

Pierre-Michel Menger

\section{OpenEdition}

\section{Journals}

Édition électronique

URL : http://journals.openedition.org/ress/412

DOI : $10.4000 /$ ress.412

ISSN : 1663-4446

Éditeur

Librairie Droz

\section{Édition imprimée}

Date de publication : 1 mars 2004

Pagination : 225-235

ISBN : 2-600-00941-8

ISSN : 0048-8046

Référence électronique

Pierre-Michel Menger, «Égalités et inégalités dans l'activité créatrice. Durkheim, Marx et Rawls devant l'individualisme artistique », Revue européenne des sciences sociales [En ligne], XLII-129 | 2004, mis en ligne le 05 novembre 2009, consulté le 30 avril 2019. URL : http://journals.openedition.org/ress/412 ; DOI : 10.4000/ress.412 
Pierre-Michel MENGER

\section{ÉGALITÉS ET INÉGALITÉS DANS L'ACTIVITÉ CRÉATRICE. DURKHEIM, MARX ET RAWLS DEVANT L'INDIVIDUALISME ARTISTIQUE}

Philippe Besnard a montré, dans son grand livre sur l'anomie (1987), que la théorie durkheimienne de l'anomie s'est construite à l'origine contre celui à qui le terme était emprunté, Guyau. Ce philosophe à qui l'on doit la première tentative nominale - de sociologie de l'art, fait de l'anomie un objectif hautement positif d'émancipation libératrice dans l'évolution humaine, i.e. le terme souhaitable, et du reste inévitable, d'un processus d'individualisation des règles morales, des croyances religieuses, des modèles comportementaux. L'activité esthétique apparaît comme une forme accomplie d'anomie positive, où l'individualisme est appelé à s'exprimer sans retenue, et qui signerait la liberté créatrice. La célébration positive de l'anomie libératrice est l'une des cibles principales de la critique en règle que Durkheim fait de la dérive anarchisante de Guyau, et, au-delà, c'est sans doute l'un des fondements durables de la méfiance de Durkheim à l'égard des pouvoirs libérateurs de l'activité esthétique, dès lors que celle-ci semble saper le ciment de l'être-ensemble social, c'est-à-dire l'appareillage moral et juridique des obligations collectives. Nous examinerons ici comment le problème de l'individualisme est abordé par Durkheim, Marx et Rawls, quand il s'incarne dans la figure et l'activité de l'artiste. Nous voulons montrer que ces trois auteurs ont en commun d'avoir vu dans l'activité de l'artiste une incarnation énigmatique, aussi troublante qu'admirée, des mouvements profonds qui agissent dans le sens de l'émancipation individualisatrice de l'être social.

\section{LES AMBIVALENCES DURKHEIMIENNES}

L'analyse durkheimienne de la régulation des différences sociales de condition et des ferments de dérèglement que recèle la dynamique sociale est connue. Les objets sur lesquels se fixent les désirs individuels intempérants sont aussi ceux-là même qui permettent l'expression la plus directe des inégalités sociales: l'imagination, se nourrissant de représentations sans cesse nouvelles d'objets désirables, trouve ses aliments dans le spectacle des consommations et des modes de vie d'autrui. Et où trouver de quoi désirer davantage sinon dans ce que détiennent ceux qui sont situés plus haut dans la hiérarchie sociale et que leur condition met en situation d'augmenter leur bien-être, leur confort, leur consommation de biens de luxe? L'imagination et l'interaction mettent ainsi en branle les pouvoirs socialement déréglants de la concurrence anomique, sur la zone de plus forte intensité concurrentielle, celle des biens d'abord réputés superflus, qu'ils soient d'une inutilité radicale ou d'une utilité encore non concevable dans les termes 
établis de la dialectique des besoins et des objets de leur satisfaction. Mais dans les deux cas, c'est pourtant le mouvement même de la civilisation et de son perfectionnement qui se fraie une voie pour enrichir et dépasser le répertoire établi des moyens (produits, procédés, pensées, pratiques, comportements) de satisfaire les fins humaines.

Or que recèlent ces activités qui sollicitent tant l'imagination et poussent à inventer du nouveau sans cesse? Elles touchent tout simplement aux fondements ultimes de la différenciation interindividuelle: une part incompressible des inégalités a, en effet, son origine dans ce que Durkheim appelle les «dons naturels » (intelligence, goût, valeur scientifique, artistique, littéraire, courage, habileté manuelle, force physique) et qu'il compare à des capitaux héréditaires : «il faudra encore une discipline morale pour faire accepter de ceux que la nature a le moins favorisés la moindre situation qu'ils doivent au hasard de leur naissance» (Le Suicide, p. 278). Ces différences de capacité et de talent doivent demeurer après la mise en œuvre d'un programme d'égalisation des «conditions extérieures de la lutte», car elles fixent les limites à placer à l'égalisation des conditions (De la division du travail social, p. 271): c'est bien là l'essence méritocratique de la régulation sociale imaginée par Durkheim.

Selon la conception durkheimienne de l'organisation optimale du travail dans les sociétés contemporaines (Lukes, 1967; Besnard, 1987), la division du travail, loin d'être une source d'aliénation, conditionne en effet l'épanouissement des individus en contraignant les désirs individuels, porteurs d'anarchie, à se soumettre à la discipline collective de la coopération interindividuelle. Pour remplir une telle fonction, la division du travail doit, dans l'idéal, se modeler «spontanément»sur les capacités différentes et particulières de chacun, de sorte que les inégalités sociales de rôle professionnel et de statut expriment exactement les inégalités dites naturelles.

L'une des limites de cette conception de l'organisation optimale du travail est son caractère statique: l'appariement maximisateur de bien-être suppose que la variété des emplois ou des tâches correspondant aux caractéristiques de chaque emploi soit suffisamment importante pour permettre aux travailleurs dotés de compétences et de préférences diverses d'opérer des choix complémentaires, $i . e$. mutuellement compatibles et individuellement satisfaisants. Chacun demeure ainsi attaché à un emploi, une fois l'appariement opéré.

Chaque travailleur est en outre supposé se connaître parfaitement et disposer d'une information parfaite sur la variété des emplois entre lesquels choisir; l'exercice du métier ne fournit à l'individu aucune information imprévue sur ses talents et ses compétences. La variété des tâches et celle des qualifications, mutuellement appariées dans un marché réalisant une division optimale du travail, contiennent d'emblée tout le potentiel de différenciation nécessaire à la maximisation du bienêtre, pour un niveau de productivité et un niveau de rémunération donnés.

A priori, les activités artistiques devraient figurer normalement parmi les incarnations du travail divisé et spécialisé auquel pourraient se consacrer ceux qui sont porteurs des talents requis. Et de fait, les «dons» artistiques figurent en bonne place dans la panoplie de ces mérites ultimement porteurs des différences interindividuelles de capacité. Mais, dans la conception durkheimienne, «l'activité esthétique » recèle beaucoup trop d'ambivalences pour ne pas être l'objet de la plus vive méfiance. 
Assurément, nulle activité humaine aussi valorisée socialement ne sollicite davantage la liberté, et d'abord la liberté de n'être asservie à aucune fin précise: c'est la leçon que Durkheim, et toute sociologie occupée à caractériser l'art par son degré d'autonomie, tire de la théorie kantienne de l'activité esthétique. Nulle activité ne porte dès lors aussi haut le pouvoir systématique de l'imagination, faculté par excellence de l'invention et du jugement esthétiques. Mais les individus trouvent, dans l'univers des produits de consommation à forte teneur d'innovation que sont les biens artistiques, les aliments symboliques par excellence de la satisfaction imaginaire de besoins qui s'étendent sans cesse et sans limite, et qui nourrissent l'intempérance individualiste. Pire encore, la consommation culturelle et artistique est un terrain particulièrement propice à l'exercice de la comparaison envieuse, celle qui empoisonne les rapports interindividuels, quand s'y expriment les inégalités de condition suscitées par les différences de classe. Car ce qui définit l'art et les activités de création et de consommation culturelle est le rejet des limites et des contraintes, et notamment des limites dans lesquelles chacun devrait contenir ses désirs et ses envies, au regard de ses capacités, sans se laisser hanter par les différences avec autrui. C'est dire si l'art attente au mécanisme pivot de l'équilibre social tel que le conçoit Durkheim:

L'art [...] est absolument réfractaire à tout ce qui ressemble à une obligation, car il est le
domaine de la liberté. C'est un luxe et une parure qu'il est peut-être beau d'avoir, mais
que l'on ne peut pas être tenu d'acquérir: ce qui est superflu ne s'impose pas. Au
contraire, la morale, c'est le minimum indispensable, le strict nécessaire, le pain quoti-
dien sans lequel les sociétés ne peuvent pas vivre. L'art répond au besoin que nous avons
de répandre notre activité sans but, pour le plaisir de la répandre, tandis que la morale
nous astreint à suivre une voie déterminée vers un but défini: qui dit obligation dit du
même coup contrainte. Ainsi, quoiqu'il puisse être animé par des idées morales ou se
trouver mêlé à l'évolution des phénomènes moraux proprement dits, l'art n'est pas moral
par soi-même. Peut-être même l'observation établirait-elle que, chez les individus,
comme dans les sociétés, un développement intempérant des facultés esthétiques est un
grave symptôme au point de vue de la moralité. (De la division du travail social, p. 14)

L'art incarne en réalité, et avec un relief tout particulier, l'ambiguïté constitutive de l'individualisme. La dimension positive du développement de l'individualisme tient au «progrès de la personnalité individuelle», avec les bienfaits sociaux qui en résultent. Le progrès de l'individualisme n'est-il pas le ressort même de l'activité artistique, puisque l'expression délibérée de la singularité individuelle constitue le vecteur de la recherche d'originalité créatrice et qu'à ce titre, elle constitue, par le jeu de la concurrence interindividuelle, le ferment de l'innovation? Durkheim ne rappelle-t-il pas à maintes reprises que sans le pouvoir de la faculté créatrice par excellence qu'est l'imagination, les individus ne seraient pas poussés à inventer sans cesse, à rechercher des solutions nouvelles pour satisfaire de nouveaux besoins, bref à progresser? Le perfectionnement civilisateur ne peut se frayer qu'ainsi un chemin pour subordonner les forces extérieures aux forces sociales, pour «renverser l'ordre naturel» et garantir l'instauration d'une liberté socialement conquise sur la nécessité contraignante des conditions naturelles circonstances, inégalités interindividuelles fondées en «nature » et transmises par la production «naturelle» de la succession des générations.

Mais la menace qu'enferme cette dynamique sociale est non moins pressante: la différenciation croissante des activités sociales fait de chaque acteur social un 
individu toujours plus autonome, et l'activité artistique ne fait qu'exacerber la tendance à la différenciation interindividuelle. L'artiste symbolise alors le risque d' «égoïsme», le désir de libre autodétermination et le refus de la contrainte collective. La méfiance durkheimienne à l'égard de l'art est clairement attachée à cette incarnation vivante du dérèglement individuel devenu profession.

\section{L'AUTORÉALISATION DE SOI ET L'ÉGALITÉ DES TALENTS CHEZ MARX}

On trouve chez Marx une conception symétrique et inverse de celle de Durkheim. La division du travail est la principale source d'aliénation de l'homme, notamment parce que cette organisation sociale du travail produit une différenciation par spécialisation mutilante des compétences, alors que le travail devrait être pour chaque homme le moyen de se réaliser dans la plénitude de son essence individuelle, conformément à un idéal d'origine aristotélicienne dont Elster (1989) fait l'essence du communisme marxien. La désutilité du travail s'évanouirait dans le contexte radicalement différent de la société communiste: le travail y deviendrait l'instrument par excellence du bien-être individuel. Mais une condition est mise à la viabilité de cette transformation: il faut neutraliser tous les ferments de concurrence interindividuelle par le marché qui pourraient provoquer la sélection différenciatrice de certaines aptitudes et avantager certains individus.

Le travail expressif, dont le travail artistique constituait, pour Marx, l'incarnation paradigmatique, ne permet à l'individu de se réaliser dans la plénitude de son humanité que si aucune mise en concurrence interindividuelle ne vient freiner le libre déploiement de tous les talents dont chacun est réputé être doté. Supprimer la concurrence de marché et supprimer le ressort des différences d'aptitude sont tout un.

L'anthropologie marxienne doit s'écarter de l'anthropologie durkheimienne tout autant que s'opposent leurs conceptions de la division du travail. Marx doit postuler l'égalité des talents et «dénaturaliser» les inégalités de capacité, qui ne seraient que le produit de la structure institutionnelle des sociétés capitalistes et précapitalistes, et en aucune façon une donnée intangible.

La solution marxienne revient à inventer une sorte d'individualisme indifférencié, et indifférent à lui-même, puisque les individus ne doivent surtout pas se comparer - s'admirer ou s'envier - afin d'éviter qu'affleurent des différences et, avec elles, des situations d'échange et de transaction fondées sur les avantages comparatifs dont chacun pourrait tirer profit pour faire ce que l'autre ne ferait pas aussi bien ou aussi volontiers, bref pour éviter qu'adviennent spécialisation et marchandisation de l'acte productif. Dès lors, ont fait remarquer G.A. Cohen (1978) et J. Elster (1989), la société ainsi organisée serait tout entière une communauté de créateurs. Chaque travailleur-créateur devrait être indifférent à une quelconque appréciation des résultats de son travail par autrui, parce que cette évaluation serait immanquablement porteuse des germes de la comparaison hiérarchisante. Chaque individu déploierait ses talents sans craindre de les voir asservis à une demande sociale: en d'autres termes, il s'agirait d'une société sans interactions ni intersubjectivité, d'une société de monades. Corrélativement, la possibilité de l'échec serait abolie, et la réussite, devenue certaine, serait le terme normal de chaque entreprise créatrice. 
Le plus sûr moyen d'obtenir que chacun soit indifférent à la valeur relative de sa performance, et ne se réjouisse que d'une performance absolue, est de postuler que tous les individus ont la même dotation en talents créateurs, ou le même potentiel de créativité, pour adopter un vocabulaire aujourd'hui très répandu. $\mathrm{Ce}$ que nous pouvons résumer par la triple hypothèse qui est au fondement d'une telle conception:

a) chaque individu dispose d'un ensemble de talents suffisamment nombreux et divers pour que toute forme de division du travail apparaisse mutilante, même lorsqu'elle est assortie d'une mobilité organisée entre des emplois variés;

b) ces talents existent en puissance et sont également répartis, mais les occasions de les actualiser sont diversement offertes. Il faut alors admettre que cette actualisation doit prendre un temps indéfini, et que chaque individu trouve dans le temps de la vie sociale suffisamment d'occasions pour actualiser tour à tour l'ensemble de ses talents;

c) l'acte productif, selon une logique typiquement hégélienne, s'apparente à un processus indéfini d'apprentissage, de découverte et d'actualisation de soi.

G.A. Cohen a fort justement identifié comme une faiblesse majeure de l'anthropologie philosophique de Marx le déni du besoin d'identification à soi et de rapport à autrui, condition de possibilité de l'organisation sociale d'une communauté. Et Elster a mis en lumière les traits utopiques et les nombreuses incohérences logiques de la conception marxienne de l'autoréalisation de soi par le travail créateur, sous les conditions - exigées par le modèle d'une société communiste d'abondance, d'égalité des talents et d'indivision du travail. Considérons, par exemple, le principe selon lequel chaque individu développera et utilisera toutes ses facultés. Si la solution marxienne était possible, ce serait résoudre d'un coup le problème de double optimalité - d'efficacité productive et de bien-être - qui est au cœur de l'éloge ou de la critique de la division du travail: l'épanouissement des forces productives par l'autoréalisation dans le travail créateur accroîtrait la productivité et créerait l'abondance en même temps que s'opérerait un transfert de bien-être des satisfactions recherchées dans la consommation vers celles procurées par le travail, qui deviendrait le «premier besoin de la vie». Cet idéal est-il plus vraisemblable que celui de l'égalité des talents naturels? La collectivité ne peut se transformer en une communauté de créateurs qu'à une condition exorbitante: que le désir d'autoréalisation de chacun ne fasse obstacle à celui de personne d'autre, ce qui requiert une société d'abondance, ou, en d'autres termes, une absence de contraintes dans la maximisation par chaque individu de son utilité.

Une autre manière d'observer l'impossible conciliation du postulat anthropologique de l'égalité des talents avec la puissance d'individuation que recèle le travail, et qui se libère dans l'autoréalisation de soi par le travail libre et créateur, est d'observer la production artistique et son développement. Jusqu'ici, dans l'histoire des arts, de diverses manières, les comparaisons hiérarchisantes et les tris sélectifs sont allées de pair avec les valeurs cardinales de l'activité - la nouveauté des œuvres et l'individualisation expressive du travail créateur. Car réussir ou échouer doivent s'entendre en deux sens : ils ne peuvent pas dépendre simplement de l'appréciation personnelle par le créateur du degré d'accomplissement de son 
travail, hors de tout repère comparatif et de toute référence à des jugements esthétiques. L'originalité, ou le manque d'originalité, de l'invention individuelle, et la valeur d'accomplissement sur un sentier d'apprentissage n'ont de sens que dans un monde de comparaison interindividuelle. Ce sont les formes de concurrence qui varient, et qui caractérisent les ressorts historiquement changeants de la formation de la valeur.

En réalité, comparaison et concurrence ne sont pas séparables de la dimension d'incertitude qui est le pivot des activités créatrices. La concurrence caractérise assurément un système de transaction et de détermination des valeurs et des prix, mais nous pouvons dire tout aussi précisément qu'elle structure la relation d'incertitude dans les interactions et les interdépendances avec autrui (pairs, professionnels du monde d'activité considérée, public). Tel est le versant stratégique de la dimension d'incertitude. L'autre versant concerne le cours même de l'activité créatrice: celle-ci n'est jamais assurée de parvenir à son terme, et c'est là ce qui rend l'accomplissement du travail créateur si désirable. L'incertitude intrinsèque est une condition nécessaire et redoutée: par elle, le travail peut être inventif, expressif, non routinier, mais par elle aussi, il constitue un défi toujours éprouvant et toujours accommodé, puisque le travail est tâtonnant, orienté vers un achèvement, mais sans terme clairement et confortablement assignable (Menger, 1989). Les régimes d'invention artistique sont, en ce sens, appariés à des régimes de gestion de l'incertitude.

\title{
ÉPANOUISSEMENT INDIVIDUEL ET BIEN-ÊTRE COMMUNAUTAIRE: L'ÉQUIVOQUE SYNTHÈSE DE RAWLS
}

La Théorie de la justice de John Rawls peut, à première vue, paraître receler les éléments d'une tentative de synthèse entre la conception durkheimienne qui affirme les inégalités de talent et la nécessité d'une division du travail, d'une part, et la conception marxienne de la réalisation de soi par l'exercice le plus développé possible de ses talents dans le travail créateur, d'autre part.

Rawls établit la rationalité de l'épanouissement individuel dans l'exercice productif de ses talents à partir du principe aristotélicien de l'accomplissement individuel sur le long terme:

\begin{abstract}
Conformément au principe aristotélicien, je suppose que les êtres humains [...] préfèrent le projet à long terme le plus complet parce que son exécution implique probablement une combinaison plus complexe de talents. Le principe aristotélicien affirme que, toutes choses égales par ailleurs, les êtres humains aiment exercer leurs talents (qu'ils soient innés ou acquis) et que plus ces talents se développent, plus ils sont complexes, plus grande est la satisfaction qu'ils procurent. On prend d'autant plus de plaisir à une activité qu'on y devient plus compétent et, entre deux activités qu'on exerce également bien, celle qu'on préfère est celle qui fait appel à une plus large gamme de jugements plus subtils et plus complexes. Ainsi le désir de réaliser le système de fins le plus large, qui met en jeu les talents les plus finement développés, est un aspect du principe aristotélicien. (op. cit., p. 455)
\end{abstract}

Mais comment, sans récuser ni les différences de talents ni la demande individuelle d'accomplissement de soi, parvenir à un équilibre de bien-être collectif à 
partir de ce principe? La solution de Rawls consiste à transformer les excellences qui résultent de différences de talent en biens collectifs, profitables pour tous, autrement dit, à «collectiviser» les biens que constituent les atouts et talents naturels de l'individu, et à les opposer ainsi aux biens et aux services de consommation qui satisfont essentiellement des préférences égoïstes.

Distinguons entre des choses qui sont bonnes en premier lieu pour nous (celui qui les
possède) et des attributs de notre personne qui sont bons à la fois pour nous et pour les
autres. [...] Ainsi, des marchandises et des biens de propriété (des biens exclusifs) sont
des biens essentiellement pour ceux qui les possèdent et les utilisent, et seulement indi-
rectement pour les autres. Par contre, l'imagination et l'esprit, la beauté et la grâce, ainsi
que d'autres atouts et talents naturels de l'individu, sont des biens aussi pour les autres:
ils sont l'objet d'une satisfaction pour nous-mêmes comme pour nos associés, s'ils se
manifestent de la bonne façon et à bon escient. Ils constituent les moyens humains pour
des activités complémentaires dans lesquelles les individus coopèrent et prennent plaisir
à la réalisation de leur propre nature ainsi qu'à celle d'autrui. Cette classe de biens
constitue les excellences [...]. Les excellences représentent les qualités et talents indivi-
duels qu'il est rationnel que chacun (y compris nous-même) désire nous voir posséder.
Ainsi, les excellences sont une condition de l'épanouissement de l'homme: elles sont des
biens pour tout le monde. Elles sont ainsi en rapport avec les conditions du respect de soi-
même, ce qui explique leur lien avec le sentiment de confiance en notre propre valeur.
(op. cit., p. 483 )

La rationalité qui émerge de cette transfiguration des talents individuels en biens collectifs et mutuellement profitables étend à tous les membres de la société l'impératif aristotélicien d'épanouissement des talents en se dotant désormais d'une contrainte sociale: un comportement purement individualiste et égocentrique d'épanouissement serait autodestructeur, puisque la singularité irréductible des talents enfouis en chaque individu ne peut s'actualiser qu'au spectacle stimulant des talents d'autrui:

Il y a aussi un principe associé du principe aristotélicien. En voyant chez les autres l'exercice de compétences de haut niveau, nous y prenons du plaisir et le désir s'éveille en nous de faire des choses semblables nous-mêmes. Nous voulons ressembler à ces individus qui ont développé des compétences que nous trouvons latentes dans notre nature. (op. cit., p. 468, souligné par nous)

Une rationalité collective émerge de l'exaltation des qualités les plus intrinsèquement personnelles:

Un projet rationnel - dans le cadre, comme toujours, des principes du juste - permet à l'individu de s'épanouir dans les limites du contexte et d'exercer ses compétences autant qu'il le peut. En outre, il est probable que ses associés soutiendront de telles activités favorables à l'intérêt commun, et qu'ils prendront du plaisir au spectacle de l'excellence humaine. Dans la mesure donc où l'on désire l'estime et l'admiration des autres, les activités favorisées par le principe aristotélicien sont également bonnes pour les autres. (op. cit., p. 469)

Comment cela se peut-il? C'est que la différence est un principe essentiel, en tant qu'elle est fondée sur la variété des talents et des compétences, et sur le fait que personne ne peut réaliser la totalité de ses talents et compétences. La question est alors: les individus ont-ils tous, en puissance, des capacités identiquement variées, mais qu'ils actualisent incomplètement, étant donné qu'ils ont des projets 
et des objectifs différents? Ou bien sont-ils réellement différents entre eux, certains disposant de compétences que d'autres n'auront jamais?

La réponse de Rawls est ambiguë. D'une part, l'horizon d'actualisation des potentialités individuelles est borné par la finitude de la vie humaine, ce qui, à soi seul, suffirait à suspendre une interrogation radicale sur l'identité de la dotation en talents qui, dans une société idéale, se déploieraient sans freins.

\begin{abstract}
Non seulement nous tirons des avantages de la nature complémentaire de nos tendances quand elles sont développées, mais encore nous prenons plaisir aux activités des autres. C'est comme si les autres faisaient apparaître une partie de nous-mêmes que nous n'aurions pas été capables de cultiver. Nous avons dû nous consacrer à autre chose qui ne représente qu'une petite partie de ce que nous aurions pu faire. (op. cit., p. 488)

Dans l'analyse du bien comme rationalité, nous étions arrivés à la conclusion bien connue que les projets rationnels de vie permettent, dans des conditions normales, le développement de certaines au moins des capacités de l'individu. C'est ce qu'indique le principe aristotélicien. Cependant, une des caractéristiques de base de l'être humain, c'est qu'il ne peut pas faire tout ce qu'il aimerait faire; ni a fortiori tout ce que quelqu'un d'autre peut faire. Les potentialités de chacun sont plus grandes que ce qu'il peut espérer réaliser; et elles sont loin d'atteindre ce qu'il est dans le pouvoir des êtres humains de faire, d'une manière générale. Ainsi, chacun doit pouvoir choisir parmi ses talents et ses intérêts possibles ceux qu'il souhaite développer; il doit en planifier l'exercice et en ordonner la pratique. (op. cit., p. 566-567)
\end{abstract}

D'autre part, le principe de différence désigne incontestablement des inégalités naturelles et sociales irréductibles qui s'expriment par des différences de productivité individuelle. Mais Rawls entend affirmer les différences sans les transformer en avantages concurrentiels générateurs d'inégalités illégitimes. Les différences ne peuvent se déployer pleinement et harmonieusement qu'en étant mises au service de tous:

Différents individus, ayant des capacités semblables ou complémentaires, peuvent coopérer en quelque sorte pour réaliser leur nature commune ou complémentaire. Quand on exerce ses propres forces en toute sécurité, on est mieux disposé à apprécier les perfections des autres, en particulier quand leurs qualités ont leur place dans une forme de vie dont les objectifs sont acceptés par tous. (ibidem)

C'est là la manifestation de ce que Dupuy (1992) a bien décrit comme l'ambivalence du système rawlsien, quand celui-ci s'attache à fonder en raison le concept de juste inégalité, ce qui lui vaut d'être pris entre deux feux critiques, contre son égalitarisme et contre sa légitimation des inégalités. Le principe de différence doit trouver son expression en même temps que son équilibre dans une société communautaire, où chacun ne se réalise qu'au contact et en relation avec autrui. Autrement dit, les talents dont chacun recherche l'épanouissement pour atteindre à l'estime de soi et au sentiment de maîtrise sont un patrimoine commun d'excellences et si les individus en sont inégalement dotés, leurs dotations différentes ne valent rien si un échange généralisé d'estime et d'admiration réciproque n'en soutient pas l'usage.

Entre les conceptions durkheimienne et marxienne de la relation entre distribution des talents et nature du travail et de ses implications collectives quant à l'accomplissement de soi dans le travail, l'entreprise rawlsienne opère une synthèse dont le caractère équivoque se manifeste bien dans la reformulation 
ambivalente de l'impératif de la division du travail, puisque tout travail serait enrichi, épanouissant et complémentaire des autres:

\begin{abstract}
Une société bien ordonnée ne supprime pas la division du travail au sens le plus général. On peut certainement dépasser les pires aspects de cette division, il n'y a pas de raison que quiconque soit servilement dépendant des autres et doive choisir des occupations monotones et routinières qui ruinent la pensée et la sensibilité humaines. On doit pouvoir offrir à chacun des tâches variées afin que les différents éléments de sa nature puissent trouver à s'exprimer. Mais si le travail devient intéressant pour tous, nous ne pouvons pas surmonter - et nous ne devrions pas le souhaiter d'ailleurs - notre dépendance à l'égard des autres[...]. Il est tentant de supposer que tous les partenaires pourraient réaliser pleinement leurs capacités et que certains, au moins, peuvent devenir des modèles achevés d'humanité. Mais c'est impossible. C'est un trait de la sociabilité humaine que nous ne pouvons réaliser par nous-mêmes qu'une partie de ce que nous pourrions être. Nous devons compter sur les autres pour réaliser les excellences que nous devons nous-mêmes laisser de côté ou qui nous font défaut. C'est l'activité collective de la société, c'est-àdire les nombreux groupes et la vie publique de la communauté la plus large qui les gouverne, qui soutient nos efforts et suscite notre contribution. Mais le bien qui est atteint par la culture collective dépasse de loin notre travail au sens où nous cessons d'être de simples fragments. La part de nous-mêmes que nous réalisons directement se relie à un système plus large et juste dont nous défendons les objectifs. La division du travail est dépassée, grâce à une activité volontaire intéressante dans le cadre d'une juste communauté de communautés à laquelle tous peuvent librement participer selon leurs tendances. (Rawls, op. cit., p. 571)
\end{abstract}

\title{
CONCLUSION
}

Sommes-nous, avec la construction à laquelle aboutit Rawls, en présence d'une synthèse efficace? Sommes-nous si loin de la position durkheimienne? La difficulté à penser ensemble la réalisation la plus complète de soi dans la différenciation et l'organisation collective d'une société viable, intégratrice, équilibrée, constitue un défi identique à celui qu'affrontait Durkheim.

L'argumentation rawlsienne se saisit des arts et des activités créatrices comme d'un ferment totalement positif parce qu'il dote l'individualisme accompli d'une sorte de principe d'autolimitation - celui de la relation heureuse à autrui, dans le creuset d'une commune humanité engagée à faire son bien collectif de toutes les capacités individuelles les plus différentes. Comment bloquer la comparaison envieuse? L'hypothèse virtualiste veut que nous portions potentiellement en nous, à l'état imparfait et imparfaitement réalisable, toutes sortes d'autrui, mais que nous n'actualisions que nous-même: l'individuation est au prix de cette imperfection sélective, mais recèle aussi le bénéfice du déploiement des différences. Moyennement un schéma initial d'égalisation des chances et des solutions redistributives, la comparaison envieuse sera, selon Rawls, contrecarrée par l'admiration de chacun pour ces biens collectifs que sont les talents différemment réalisés en tous les membres d'une société.

Marx avait radicalisé le raisonnement: chacun porte en lui toutes les qualités, talents et capacités qui peuvent le faire ressembler à tout autrui possible, et un monde d'abondance permettrait à ce potentiel illimité et indéfiniment actualisable de se réaliser sans conflit interindividuel. Mais le raisonnement marxien est doublement problématique: il suppose réalisée une suppression parfaite des 
contraintes de rareté des ressources, et il postulait l'accomplissement parfait de soi, égalitaire par indifférenciation et par impossibilité de l'échec.

Durkheim multiplie les raisonnements à double fond. Tantôt il pose le primat logique et sociologique de la vie collective, contre les théoriciens de l'individualisme qui, tel Spencer, déduisent la société de l'individu. Dans ce cas, la différenciation des individus et le déploiement de leurs aptitudes différenciées dans l'espace du travail socialement divisé procèdent de l'évolution d'une vie toujours déjà collective (voir par exemple, De la division du travail social, p. 261): les progrès de la division du travail ne font qu'amplifier l'intensité et la variété prises par cette différenciation foncièrement socialisée, selon un mécanisme d'autorenforcement abondamment rappelé. Tantôt le vocabulaire adopté par Durkheim est virtualiste, pour suggérer que des potentialités resteraient sommeillantes, enveloppées, si elles n'étaient pas sollicitées par les circonstances extérieures de se développer.

Tout se passe mécaniquement: une rupture d'équilibre dans la masse sociale suscite des conflits qui ne peuvent être résolus que par une division du travail plus développée. Quant aux circonstances extérieures, aux combinaisons variées de l'hérédité, comme les déclivités du terrain déterminent la direction du courant, mais ne le créent pas, elles marquent le sens dans lequel se fait la spécialisation là où elle est nécessaire, mais ne la nécessitent pas. Les différences individuelles qu'elles produisent resteraient à l'état de virtualité si, pour faire face à des difficultés nouvelles, nous n'étions contraints de les mettre en saillie et de les développer. (De la division du travail social, p. 252)

Nous retrouvons ici les ambiguïtés caractéristiques de toute construction dualiste quand elle est soumise à un traitement argumentatif à la fois logique et généalogique: il faut à la fois affirmer l'unité d'une origine (le primat du collectif) et loger un principe de différenciation qui sera mis en branle par des circonstances extérieures, donc fixer une origine logique et l'affecter d'emblée d'impureté logique, pour que l'histoire puisse se produire. Car l'histoire, dans la construction durkheimienne, n'est que la somme des écarts cumulatifs et exponentiels à l'équilibre: équilibre entre besoins et milieu, équilibre entre fonctions et capacités, équilibre entre spécialisation croissante des fonctions et solidarité interindividuelle, équilibre entre transmission héréditaire de dotations individuelles génératrices d'inégalités a priori et exigence d'équité sociale ou, en d'autres termes, typiquement durkheimiens, équilibre entre inégalités «intérieures» (car fondées en nature) au groupe social et égalité des conditions «extérieures» de la lutte sociale.

Empire des différences et des individualismes, domaine par excellence de l'affranchissement à l'égard des règles, mais aussi territoire de l'exploration sans fin des ressources de l'imagination créatrice, l'art adresse à Durkheim un défi que l'on résumera aisément dans l'équation sociale dont Philippe Besnard a si magistralement identifié les résolutions partielles chez Durkheim, l'équation qui doit associer la différenciation individualisatrice des sociétés organiques aux exigences intégratrices de correction et de limitation des différences et inégalités de capacité dont pourtant l'évolution des sociétés fait l'un des carburants de son développement.

Centre de Sociologie du Travail et des Arts (CESTA)

École des Hautes Études en Sciences Sociales, Paris menger@ehess 


\section{RÉFÉRENCES BIBLIOGRAPHIQUES}

Besnard Ph., 1987, L'anomie, ses usages et ses fonctions dans la discipline sociologique depuis Durkheim, Paris, Puf.

Cohen G. A., 1978, Karl Marx's Theory of History, Oxford, Oxford UP.

Dupuy J.-P., 1992, Le sacrifice et l' envie, Paris, Calmann-Lévy.

Durkheim É., 1960[1893], De la division du travail social, Paris, Puf.

Elster J., 1989, Karl Marx. Une interprétation analytique, Paris, Puf.

Lukes S., 1967, «Alienation and Anomie», in P. Laslett, W. Runciman (eds.), Philosophy, Politics and Society, Oxford, Blackwell, vol. 3, 134-156.

Menger P.-M., 1989, «Rationalité et incertitude de la vie d'artiste», L'Année sociologique, 39, 111151.

Rawls J., 1987, Théorie de la justice, Paris, Seuil. 